\title{
A Survey of the Implementation of Numerical Schemes for Linear Advection Equation
}

\author{
Pedro Pablo Cárdenas Alzate \\ Department of Mathematics, Universidad Tecnológica de Pereira, Pereira, Colombia \\ Email: ppablo@utp.edu.co
}

Received 16 June 2014; revised 16 July 2014; accepted 3 August 2014

Copyright (C) 2014 by author and Scientific Research Publishing Inc.

This work is licensed under the Creative Commons Attribution International License (CC BY).

http://creativecommons.org/licenses/by/4.0/

(c) (i) Open Access

\begin{abstract}
The interpolation method in a semi-Lagrangian scheme is decisive to its performance. Given the number of grid points one is considering to use for the interpolation, it does not necessarily follow that maximum formal accuracy should give the best results. For the advection equation, the driving force of this method is the method of the characteristics, which accounts for the flow of information in the model equation. This leads naturally to an interpolation problem since the foot point is not in general located on a grid point. We use another interpolation scheme that will allow achieving the high order for the box initial condition.
\end{abstract}

\section{Keywords}

Numerical Schemes, Advection Equation, Semi-Lagrangian Approach

\section{Introduction}

The classical 1D linear advection equation is given by

$$
u_{t}+\alpha u_{x}=0
$$

We can see that Equation (1) is a 1-D version (linear) of the partial differential equations [1], which describe advection of quantities such as energy, mass, heat, etc. Here, $u=u(x, t), \quad x \in \mathbb{R}$ and $\alpha \neq 0$ is a nonzero constant velocity. We can say that Equation (1) describes the motion of a scalar $u$ as it is advected by a known velocity field.

We know that the unique solution of Equation (1) is determined by an initial condition $u_{0}:=u(x, 0)$ where $u(x, t)=u_{0}(x-\alpha t)$ with $u_{0}$ an arbitrary function on $\mathbb{R}$. 
An analysis of a numerical scheme implies to study the consistency, stability and accuracy. The goal of the analysis is to get an idea about how good a difference scheme is representing the linear advection equation. In the following we will make some analysis of the approximate difference schemes to the Equation (1).

Numerical problems arising with pollutions models are discussed in [2]. Textbooks that deal with advection problems are [3] and [4] The spectral-methods and finite elements for linear advection equations we refer are [5] and [6] respectively. There exists more recent bibliography on finite element methods, which can be consulted in [7].

The paper is organized as follows. In Sections 2 and 3, we consider two cases firstly $u_{0}=\cos (x)$ and here we study the implementation of the schemes Lax-Friedrich, Lax-Wendroff and RK3-TVD-WENO5 and we derive the expected GTE (Global Truncation Error) and we verify it by producing a convergence plot (by using the $l_{1}, l_{2}$ and $l_{\infty}$ norm). After this, we repeat by using the box function

$$
u_{0}= \begin{cases}1, & x<\frac{\pi}{4} \\ 2, & \frac{\pi}{4} \leq x<\frac{\pi}{2} \\ 1, & x \geq \frac{\pi}{2}\end{cases}
$$

In Section 4, we consider linear and quadratic interpolation and we study the stability and accuracy by using semi-Lagrangian approach for linear advection equation and finally we produce a convergence plot for (2) with another interpolation scheme.

\section{Implementation of the Schemes and GTE}

Initially we consider the 1-D linear advection equation

$$
\begin{gathered}
u_{t}+u_{x}=0, \quad x \in S^{1}, \quad t \in(0, T] \\
u(x, 0)=u_{0}(x)
\end{gathered}
$$

We can to verify that the solution is $u(x, t)=u_{0}(x-t)$, and therefore for $T=1 u(x, 1)=u_{0}(x-1)$ [8]. Now, we compute the global truncation error for the schemes as follows.

1) Lax-Friedrich. Here, the discretization of the advection equation by using Lax-Friedrich gives

$$
\frac{u_{j}^{n+1}-\frac{1}{2}\left(u_{j+1}^{n}+u_{j-1}^{n}\right)}{\Delta t}=-\frac{u_{j+1}^{n}-u_{j-1}^{n}}{2 \Delta x}
$$

and therefore we can verify

$$
\begin{aligned}
\xi(\Delta x, \Delta t) & =\frac{u(x, t+\Delta t)-\frac{1}{2}(u(x+\Delta x, t)+u(x-\Delta x, t))}{\Delta t}+\frac{u(x+\Delta x, t)-u(x-\Delta x, t)}{2 \Delta x} \\
& =O\left(\Delta t+(\Delta x)^{2}\right)
\end{aligned}
$$

so the stability condition is

finally

$$
\frac{\Delta t}{\Delta x} \leq 1 \Leftrightarrow t=K(\Delta x)
$$

$$
\mathrm{GTE}_{\mathrm{L}-\mathrm{F}} \leq \xi(\Delta x, \Delta t)=O\left(\Delta t+(\Delta x)^{2}\right)=O(\Delta x)
$$

2) Lax-Wendroff. Here, the discretization of the advection equation by using Lax-Wendroff gives

$$
\frac{u_{j}^{n+1}-u_{j-1}^{n}}{\Delta t}=-\frac{u_{j+1}^{n}-u_{j-1}^{n}}{2 \Delta x}+\left(\frac{\Delta t}{2}\right) \frac{u_{j+1}^{n}-2 u_{j}^{n}+u_{j-1}^{n}}{(\Delta x)^{2}}
$$


and therefore we can verify

$$
\begin{aligned}
\xi(\Delta x, \Delta t)= & \frac{u(x, t+\Delta t)-u(x, t)}{\Delta t}+\frac{u(x+\Delta x, t)-u(x-\Delta x, t)}{2 \Delta x} \\
& -\left(\frac{\Delta t}{2}\right) \frac{u(x+\Delta x, t)-2 u(x, t)+u(x-\Delta x, t)}{(\Delta x)^{2}} \\
= & O\left((\Delta t)^{2}-(\Delta x)^{2}\right) .
\end{aligned}
$$

and the stability condition (CFL) is

$$
\frac{\Delta t}{\Delta x} \leq 1 \Leftrightarrow t=K(\Delta x)
$$

Therefore,

$$
\operatorname{GTE}_{\mathrm{L}-\mathrm{W}} \leq \xi(\Delta x, \Delta t)=O\left((\Delta x)^{2}+(\Delta x)^{2}\right)=O\left((\Delta x)^{2}\right)
$$

3) RK3-TVD + WENO5. For this scheme the one-step is

$$
\sigma(\Delta t, \Delta x)=O\left((\Delta t)^{4}+(\Delta x)^{6}\right)
$$

and the stability condition is

$$
\frac{\Delta t}{\Delta x} \leq 1 \Leftrightarrow t=K(\Delta x)
$$

therefore,

$$
\mathrm{GTE}_{\mathrm{R} 3 \mathrm{~W} 5} \leq \frac{1}{\Delta x} \xi(\Delta x, \Delta t)=\frac{1}{\Delta x} O\left((\Delta x)^{4}+(\Delta x)^{6}\right)=O\left((\Delta x)^{3}\right)
$$

Now, for all schemes we proceed by to build the spatial grid by letting $d x=\frac{2 \pi}{2^{k}}, k \in \mathbb{Z}$ and we define the vector

$$
\begin{aligned}
X & =\{0, \mathrm{~d} x, 2 \mathrm{~d} x, \cdots,(N-1) \mathrm{d} x=2 \pi\} \\
& =\{X(1), X(2), \cdots, X(N-1), X(N)\}
\end{aligned}
$$

which has $N$ points. As the boundary conditions are periodic, we will have to make the following identifications,

$$
\cdots X(-2)=X(N-3), X(-1)=X(N-2), X(0)=X(N-1), X(1)=X(N), X(N+1)=X(2) \cdots
$$

To build the grid in time, we first $\mathrm{d} t=r \mathrm{~d} x$ for some $r$ satisfying the CFL (condition). Therefore we define $N=\operatorname{ceil}(\tau / \mathrm{d} t)$ where $\tau$ is the final time. So we going to iterate until the time $\tilde{t}=N \cdot \mathrm{d} t$. Then, in general $\tilde{t} \neq \tau$ but at least. Here, all times steps are the same size $\mathrm{d} t$. Finally, when computing the global truncation error, the only important thing is to always compute it at the same time $\tilde{t}$ for all grid resolutions. Now, the scheme (LF, LW or RK3-WENO5) is applied until $\tilde{t}$ is reached. Then the output is $u_{\text {app }}$. The grid resolution $\mathrm{d} x$, calculate what the true solution is at time $\tilde{t}$ and compute the norm of the errors by using

$$
\begin{gathered}
E_{\infty}=\max _{i=1, \cdots, K}\left|u_{\text {app }}(i)-u_{\text {true }}(i)\right| \\
E_{1}=\mathrm{d} x \cdot \sum_{i=1}^{K}\left|u_{\text {app }}(i)-u_{\text {true }}(i)\right| \\
E_{2}=\left(\mathrm{d} x \cdot \sum_{i=1}^{K}\left|u_{\text {app }}(i)-u_{\text {true }}(i)\right|^{2}\right)^{1 / 2}
\end{gathered}
$$

Here we iterate over the resolution by varying $k$ in order to get a convergence plot of the various norms of the 
errors vs. grid size.

Lax-Friedrich. This scheme can be coded very efficiently using matrices. We can see that the scheme can be rewritten as

$$
u_{j}^{n+1}=\frac{1}{2}\left[\left(1-\frac{\Delta t}{\Delta x}\right) u_{j+1}^{n}+\left(1+\frac{\Delta t}{\Delta x}\right) u_{j-1}^{n}\right]=\frac{1}{2}\left[(1-r) u_{j+1}^{n}+(1+r) u_{j-1}^{n}\right]
$$

therefore taking into account the periodic boundary conditions

$$
\left(\begin{array}{c}
u_{1} \\
u_{2} \\
u_{3} \\
\vdots \\
u_{K-2} \\
u_{K-1} \\
u_{K}
\end{array}\right)^{n+1}=\frac{1}{2}\left(\begin{array}{ccccccc}
0 & 0 & 0 & \cdots & 0 & 1-r & 0 \\
1-r & 0 & 1+r & \cdots & 0 & 0 & 0 \\
0 & 1-r & 0 & \cdots & 0 & 0 & 0 \\
\vdots & \vdots & \vdots & \ddots & \vdots & \vdots & \vdots \\
0 & 0 & 0 & \cdots & 0 & 1+r & 0 \\
0 & 0 & 0 & \cdots & 1-r & 0 & 1+r \\
0 & 1+r & 0 & \cdots & 0 & 1-r & 0
\end{array}\right)\left(\begin{array}{c}
u_{1} \\
u_{2} \\
u_{3} \\
\vdots \\
u_{K-2} \\
u_{K-1} \\
u_{K}
\end{array}\right)^{n}
$$

Lax-Wendroff. Similarly this scheme can be implemented by using

$$
\left(\begin{array}{c}
u_{1} \\
u_{2} \\
u_{3} \\
\vdots \\
u_{K-2} \\
u_{K-1} \\
u_{K}
\end{array}\right)^{n+1}=\frac{1}{2}\left(\begin{array}{ccccccc}
1-r^{2} & -\frac{r}{2}(1-r) & 0 & \cdots & 0 & \frac{r}{2}(1+r) & 0 \\
\frac{r}{2}(1+r) & 1-r^{2} & -\frac{r}{2}(1-r) & \cdots & 0 & 0 & 0 \\
0 & \frac{r}{2}(1+r) & 1-r^{2} & \cdots & 0 & 0 & 0 \\
0 & \vdots & \vdots & \ddots & \vdots & \vdots & \vdots \\
0 & 0 & 0 & \cdots & 0 & 0 & 0 \\
0 & -\frac{r}{2}(1-r) & 0 & \cdots & 0 & \frac{r}{2}(1+r) & 1-r^{2}
\end{array}\right)\left(\begin{array}{c}
u_{1} \\
u_{2} \\
u_{3} \\
\vdots \\
u_{K-2} \\
u_{K-1} \\
u_{K}
\end{array}\right)^{n}
$$

RK3-TVD-WENO5. For each point on the grid, we need to compute two intermediate values as follows.

$$
\begin{gathered}
\xi_{i}^{*}=\operatorname{FE}\left(\xi_{i}^{n}\right)=\xi_{i}^{n}-\Delta t \cdot \operatorname{WENO}\left(\xi_{i}^{n}\right) \\
\xi_{i}^{* *}=\frac{1}{4} \mathrm{FE}\left(\operatorname{FE}\left(\xi_{i}^{n}\right)\right)+\frac{3}{4} \xi_{i}^{n}=\frac{1}{4}\left(\xi_{i}^{*}-\Delta t \cdot \operatorname{WENO}\left(\xi_{i}^{*}\right)\right)+\frac{3}{4} \xi_{i}^{n} \\
\xi_{i}^{n+1}=\frac{2}{3} \mathrm{FE}\left(\xi_{i}^{* *}\right)+\frac{1}{3} \xi_{i}^{n}=\frac{2}{3}\left(\xi_{i}^{* *}-\Delta t \cdot \operatorname{WENO}\left(\xi_{i}^{* *}\right)\right)+\frac{1}{3} \xi_{i}^{n}
\end{gathered}
$$

Therefore, the code for WENO5, when solving a conservative equation of the form

$$
u_{t}+f(u)_{x}=0 \Leftrightarrow u_{t}+f^{\prime}(u) u_{x}=0
$$

provided $u$ and $f$ are $C^{1}$. Then $c(u(x, t)):=f^{\prime}(u)(x, t)$ acts as the speed function. Then at the interior point $x_{i}$. If $c\left(u_{i}^{n}\right)>0$, define

$$
\left(v_{1}, v_{2}, \cdots, v_{5}\right)=\frac{1}{\Delta x}\left(u_{i-2}-u_{i-3}, u_{i-1}-u_{i-2}, \cdots, u_{i+2}-u_{i+1}\right)
$$

We can say that $\epsilon=10^{-6}$, then

$$
s_{1}=\frac{13}{12}\left(v_{1}-2 v_{2}+v_{3}\right)^{2}+\frac{1}{4}\left(v_{1}-4 v_{2}+3 v_{3}\right)^{2}
$$




$$
\begin{gathered}
s_{2}=\frac{13}{12}\left(v_{1}-2 v_{2}+v_{4}\right)^{2}+\frac{1}{4}\left(v_{1}-v_{4}\right)^{2} \\
s_{3}=\frac{13}{12}\left(v_{3}-2 v_{4}+v_{5}\right)^{2}+\frac{1}{4}\left(3 v_{3}-4 v_{4}+v_{5}\right)^{2} \\
\left(\alpha_{1}, \alpha_{2}, \alpha_{3}\right)=\frac{1}{10}\left(\frac{1}{\left(\epsilon+s_{1}\right)^{2}}, \frac{6}{\left(\epsilon+s_{2}\right)^{2}}, \frac{3}{\left(\epsilon+s_{3}\right)^{2}}\right) \\
s_{\alpha}=\alpha_{1}+\alpha_{2}+\alpha_{3} \\
\left(w_{1}, w_{2}, w_{3}\right)=\frac{1}{s_{\alpha}}\left(\alpha_{1}, \alpha_{2}, \alpha_{3}\right)
\end{gathered}
$$

finally we obtain

$$
\operatorname{WENO}\left(u_{i}^{n}\right)=\frac{1}{6}\left[w_{1}\left(2 v_{1}-7 v_{2}+11 v_{3}\right)+w_{2}\left(-v_{2}+5 v_{3}+2 v_{4}\right)+w_{3}\left(2 v_{3}+5 v_{4}-v_{5}\right)\right]
$$

\section{Test Problems}

Example 1. We consider the condition $u_{0}(x)=\cos x$, then by using the schemes we have:

- Lax-Friedrich. The convergence results are shows on Figure 1. (Convergence plot for $u(x, t)=\cos (x-t)$ in Log-Log scale, advected by using L-F scheme. $\log (\mathrm{GTE})$ at $T \approx 1$ is plotted against $\log (h)$.) The grid resolution was varied from $\mathrm{d} x=2 \pi / 2^{5}$ to $x=2 \pi / 2^{16}$. So the expected orders of convergence were already visible at those resolutions.

- Lax-Wendroff. The convergence results are shown on Figure 2. (Convergence plot for $u(x, t)=\cos (x-t)$ in Log-Log scale, advected by using L-W scheme. $\log (\mathrm{GTE})$ at $T \approx 1$ is plotted against $\log (h)$.) As above, the grid resolution was varied from $\mathrm{d} x=2 \pi / 2^{5}$ to $x=2 \pi / 2^{11}$.

- RK3-TVD + WENO5. The convergence results are shown on Figure 3. (Convergence plot for $u(x, t)=\cos (x-t)$ in Log-Log scale, advected by using L-W scheme. $\log (\mathrm{GTE})$ at $T \approx 1$ is plotted against $\log (h)$.) As above, the grid resolution was varied from $\mathrm{d} x=2 \pi / 2^{4}$ to $x=2 \pi / 2^{11}$.

The norms errors behave as expected in the asymptotic behavior. This example underlines the importance of letting the error go to $\epsilon_{\text {machine }}$ whenever possible. Here, since the computer time became unreasonably long for

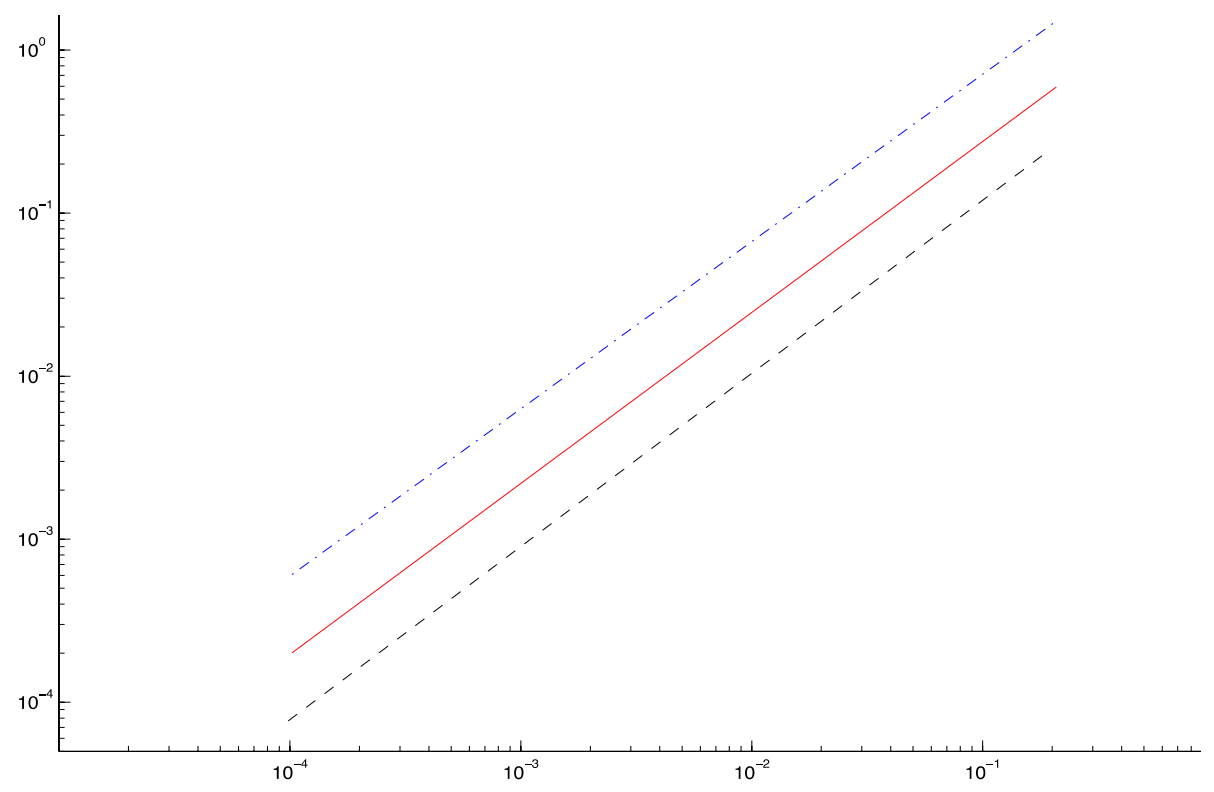

Figure 1. Convergence plot for $u(x, t)=\cos (x-t)$ with L-F scheme. 


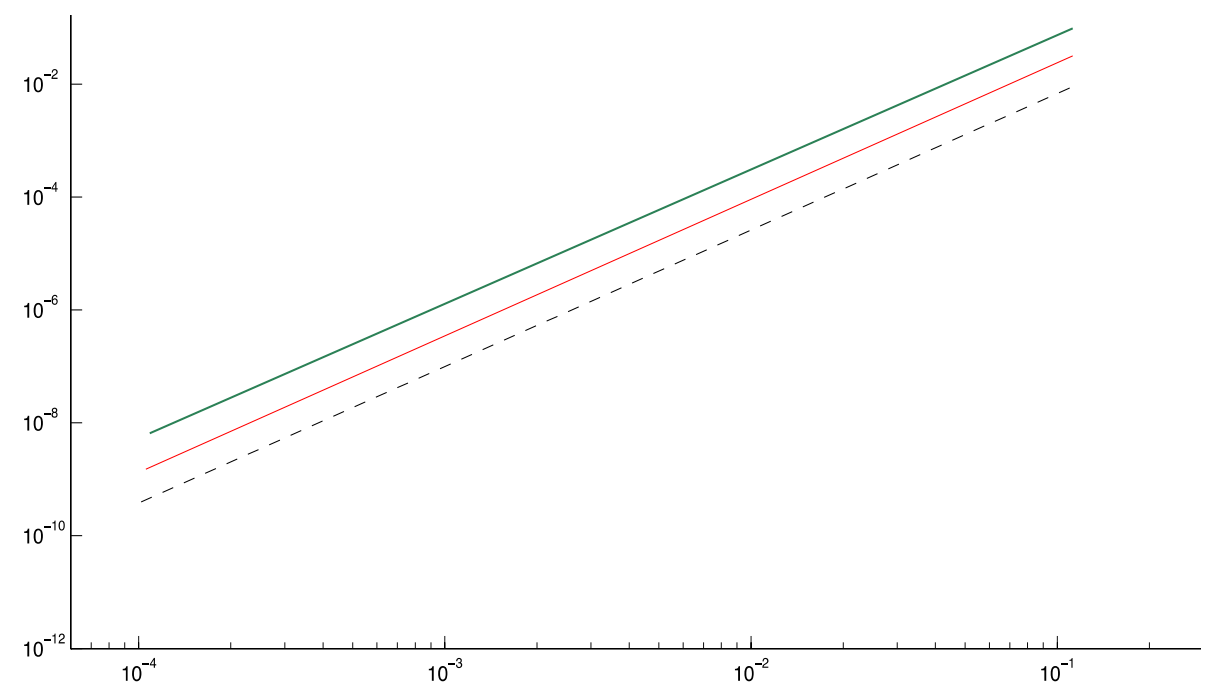

Figure 2. Convergence plot for $u(x, t)=\cos (x-t)$ with L-W scheme.

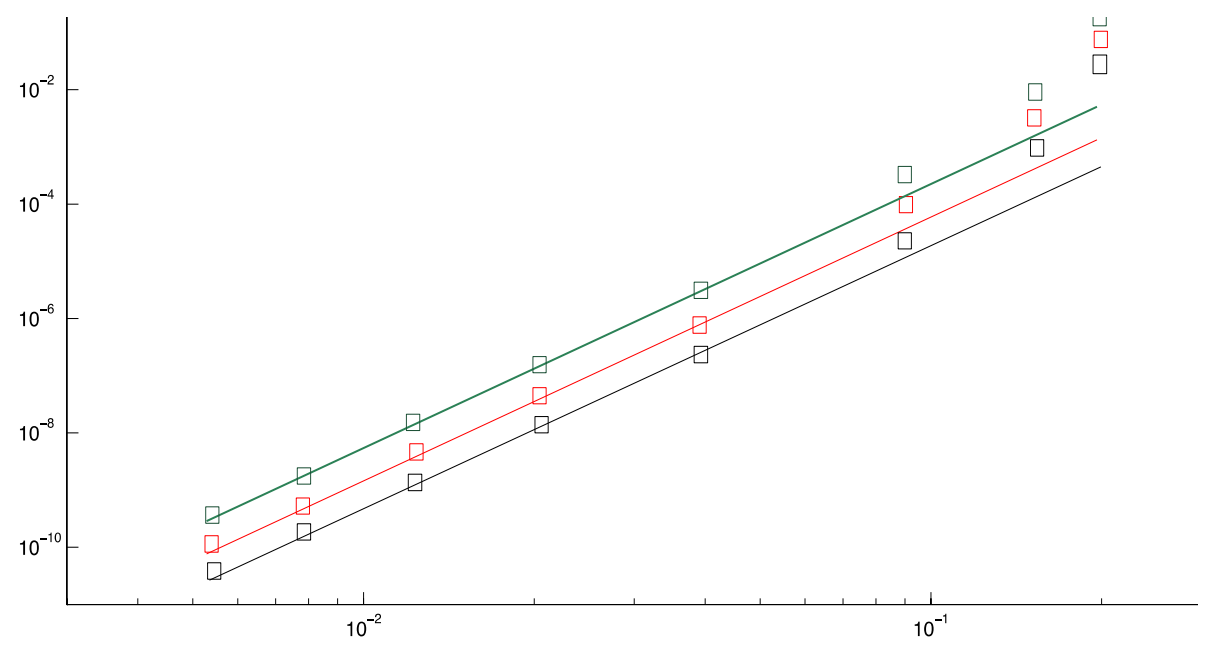

Figure 3. Convergence plot for $u(x, t)=\cos (x-t)$ with RK3-TVD-WENO5 scheme.

high grid resolutions, this was not possible. However, as suggested in class, one way to observe the order of the global truncation error (GTE) is to increase the final time (for example $T=5$ ) in order to let error in $\Delta t$ accumulate and become more significant than the error in $\Delta x$.

Example 2. Consider the box condition (2), then we have

- Lax-Friedrich. The convergence results are shown on Figure 4. (Convergence plot for $u(x, t)=\cos (x-t)$ in Log-Log scale, advected by using L-W scheme. $\log (\mathrm{GTE})$ at $T \approx 1$ is plotted against $\log (h)$.) As above, the grid resolution was varied from $\mathrm{d} x=2 \pi / 2^{4}$ to $x=2 \pi / 2^{16}$.

Therefore, since in deriving the LTE and GTE, we used the Taylor series of the function $u(x, t)$. But the box function being only $C^{0}$, we cannot expect $u(x, t)$ to equal its Taylor series point wise, and thereforethe above reasoning does not apply. It is interesting to watch the box evolve when advected with a L-F scheme. This is presented in a series of snapshots put together in Figure 5. (The true solution is plotted in red and the numerical solution is plotted in blue.) Clearly, the schemes fails to capture the discontinuities of the function, and its diffusive nature eventually leads to large errors near the discontinuities. This explains why the $L^{\infty}$ norm of the error cannot converge. This also explains why the $L^{1}$ norm, which is simply the area between the true solution and the numerical solution, improves with the grid resolution.

- Lax-Wendroff. The convergence results are shown on Figure 6. (Convergence plot for $u(x, t)=\operatorname{box}(x, t)$ 


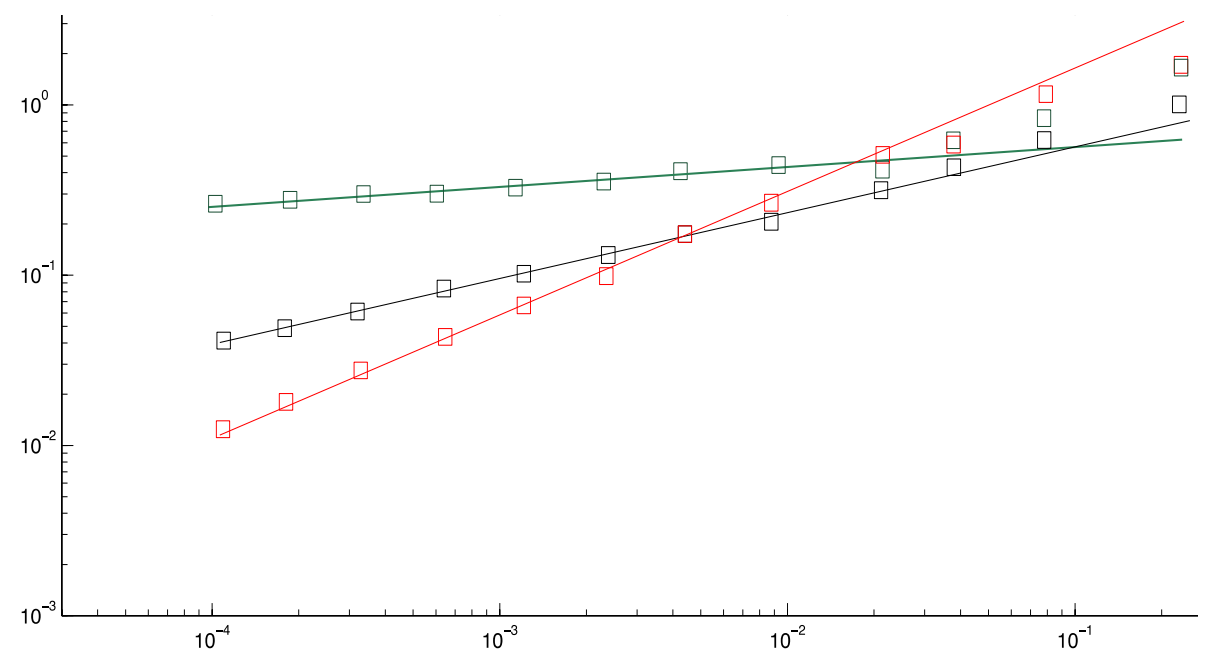

Figure 4. Convergence plot for $u(x, t)=\operatorname{box}(x, t)$ with L-F scheme.

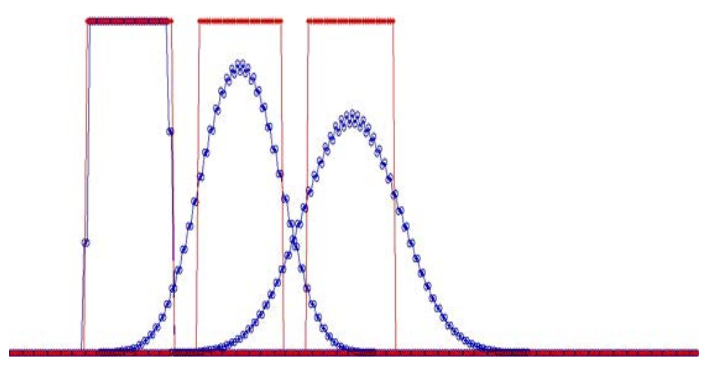

Figure 5. Snapshots for $u(x, t)=\operatorname{box}(x, t)$ with L-F scheme.

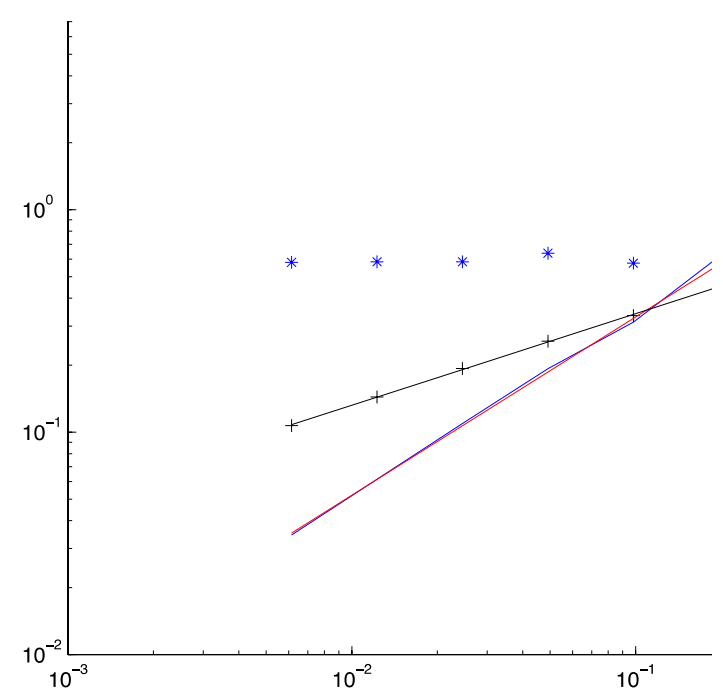

Figure 6. Convergence plot for $u(x, t)=\operatorname{box}(x, t)$ with L-W scheme.

in Log-Log scale, advected by using L-W scheme. $\log ($ GTE $)$ at $T \approx 1$ is plotted against $\log (h)$ ). As above, the grid resolution was varied from $\mathrm{d} x=2 \pi / 2^{5}$ to $x=2 \pi / 2^{16}$. The slope for the $L^{\infty}$, since there clearly isn't convergence. 
It is interesting to watch the box evolve when advected by a L-W scheme. This is presented in a series of snapshots put together in Figure 7 (true solution in red and numerical solution in blue). This scheme also fails to capture the discontinuities of the function, and its dispersive nature eventually leads to large errors near the discontinuities, where wild oscillations appear. However, the overall shape of the box is better preserved than when advected with L-F, which provides a simple explanation as to why the rate of convergence of the $L^{1}$ norm is slightly better.

- RK3-TVD-WENO5. The convergence results are shown on Figure 8. (Convergence plot for box in log-log scale, advected by using RK3-TVD-WENO5 scheme.) As above, the grid resolution was varied from $\mathrm{d} x=2 \pi / 2^{5}$ to $\mathrm{d} x=2 \pi / 2^{12}$ The final time was increased to $T=4$. Therefore

We almost get first order convergence in the $L^{1}$ norm, which is much better than with the previous two schemes. However, convergence is slower in the $L^{2}$ norm and absent in the $L^{\infty}$ norm.

Again, it is interesting to watch the box evolve when advected by an RK3-TVD-WENO5 scheme. Although this scheme also fails to capture the discontinuities of the function, the overall shape is much better preserved than when advected with the previous two linear schemes. Although the $L^{\infty}$ norm cannot converge because of the large errors near the discontinuities, those errors do not spread out and pollute the solution, which explains why the rate of convergence of the $L^{1}$ norm is almost 1 .

\section{Semi-Lagrangian Approach}

The idea is to use various values of $u_{j}^{0}$ to build an interpolant $L(x)$ and the get $u_{2}^{1}$ from $u_{2}^{1}=L\left(x_{2}-c \Delta t\right)$. We use the formula for Lagrange interpolation in each case, here $r=0.5$ and $c=1$. For the linear interpolation we use the two neighboring points $\left(x_{1}, u_{1}^{0}\right)$ and $\left(x_{2}, u_{2}^{0}\right)$, therefore
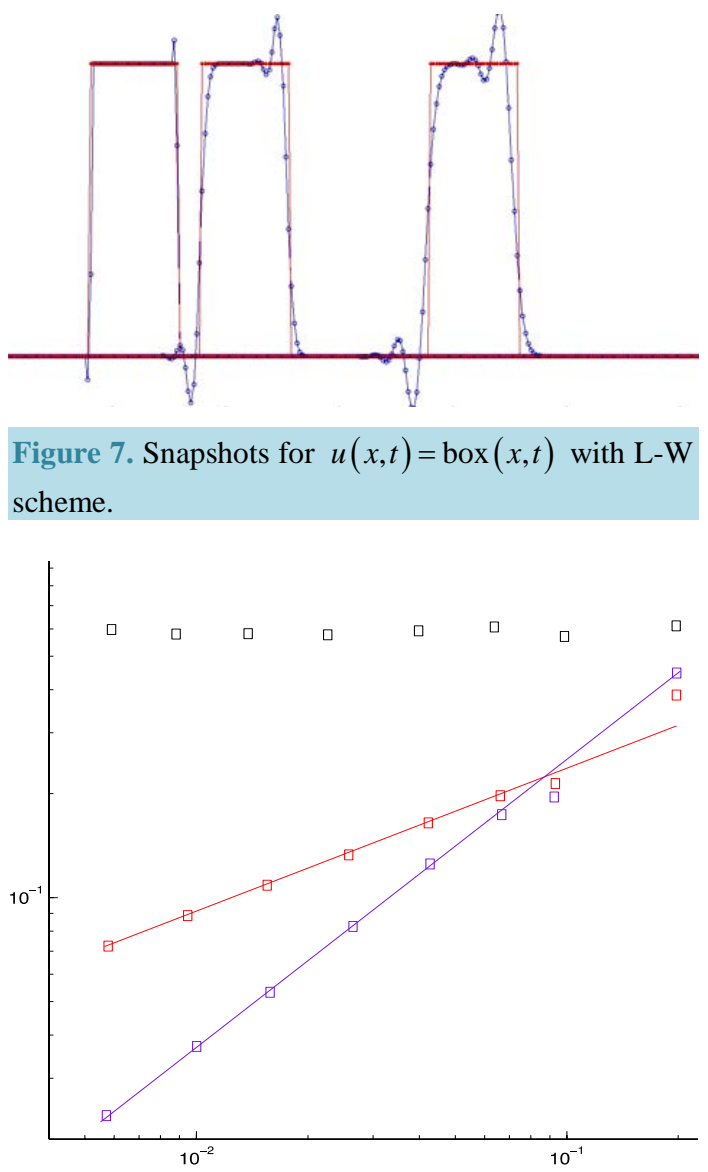

Figure 8. Convergence plot for $u(x, t)=\operatorname{box}(x, t)$ with RK3-TVD-WENO5 scheme. 


$$
L(x)=u_{1}^{0} l_{1}(x)+u_{2}^{0} l_{2}(x)=u_{1}^{0} \frac{x-x_{2}}{x_{1}-x_{2}}+u_{2}^{0} \frac{x-x_{1}}{x_{2}-x_{1}}=u_{1}^{0} \frac{x-x_{2}}{-\Delta x}+u_{2}^{0} \frac{x-x_{1}}{\Delta x}
$$

then

$$
u_{2}^{1} \approx L\left(x_{2}-c \Delta t\right)=u_{1}^{0} \frac{-c \Delta t}{-\Delta x}+u_{2}^{0} \frac{x_{2}-x_{1}-c \Delta t}{\Delta x}=u_{1}^{0} \frac{c \Delta t}{\Delta x}+u_{1}^{0}\left(1-\frac{c \Delta t}{\Delta x}\right)
$$

and finally

$$
\frac{u_{2}^{1}-u_{2}^{0}}{\Delta t}=c \frac{u_{1}^{0}-u_{2}^{0}}{\Delta x} \text { (upwind method) }
$$

We can see that if we let $r=0.5$ and $c=1$, then $u_{2}^{1}-u_{2}^{0}=-\frac{u_{2}^{0}-u_{1}^{0}}{2}$. Now, for the quadratic interpolationleft, using $\left(x_{0}, u_{0}^{0}\right),\left(x_{1}, u_{1}^{0}\right)$ and $\left(x_{2}, u_{2}^{0}\right)$ we have

$$
L(x)=u_{0}^{0} l_{0}(x)+u_{1}^{0} l_{1}(x)+u_{2}^{0} l_{2}(x)=u_{0}^{0} \frac{\left(x-x_{1}\right)\left(x-x_{2}\right)}{2 \Delta x^{2}}+u_{1}^{0} \frac{\left(x-x_{0}\right)\left(x-x_{2}\right)}{-\Delta x^{2}}+u_{2}^{0} \frac{\left(x-x_{0}\right)\left(x-x_{1}\right)}{2 \Delta x^{2}}
$$

then

$$
u_{2}^{1} \approx L\left(x_{2}-c \Delta t\right)=u_{2}^{0}+\frac{1}{\Delta x^{2}}\left[c \Delta x \Delta t\left(-u_{0}^{0}+4 u_{1}^{0}-3 u_{2}^{0}\right)+c^{2} \Delta t^{2}\left(u_{0}^{0}-2 u_{1}^{0}+u_{2}^{0}\right)\right]
$$

finally

$$
\left.\frac{u_{2}^{1}-u_{2}^{0}}{\Delta t}=-\frac{c}{2 \Delta x}\left(u_{0}^{0}-4 u_{1}^{0}+3 u_{2}^{0}\right)+\frac{c^{2} \Delta t}{2 \Delta x^{2}}\left(u_{0}^{0}-2 u_{1}^{0}+u_{2}^{0}\right) \quad \text { (Beam-Warming method }\right)
$$

Here, if we let $r=0.5$ and $c=1$, then $u_{2}^{1}-u_{2}^{0}=-\frac{1}{4}\left(u_{0}^{0}-4 u_{1}^{0}+3 u_{2}^{0}\right)+\frac{1}{8}\left(u_{0}^{0}-2 u_{1}^{0}+u_{2}^{0}\right)$ Now, with quadratic interpolation-right and by using $\left(x_{1}, u_{1}^{0}\right),\left(x_{2}, u_{2}^{0}\right)$ and $\left(x_{3}, u_{3}^{0}\right)$ we have

$$
L(x)=u_{1}^{0} l_{1}(x)+u_{2}^{0} l_{2}(x)+u_{3}^{0} l_{3}(x)=u_{1}^{0} \frac{\left(x-x_{2}\right)\left(x-x_{3}\right)}{2 \Delta x^{2}}+u_{2}^{0} \frac{\left(x-x_{1}\right)\left(x-x_{3}\right)}{-\Delta x^{2}}+u_{3}^{0} \frac{\left(x-x_{1}\right)\left(x-x_{2}\right)}{2 \Delta x^{2}}
$$

therefore

finally we have

$$
u_{2}^{1} \approx L\left(x_{2}-c \Delta t\right)=u_{2}^{0}+\frac{c \Delta t}{2 \Delta x}\left(u_{1}^{0}-u_{3}^{0}\right)+\frac{c^{2} \Delta t^{2}}{2 \Delta x^{2}}\left(u_{3}^{0}-2 u_{2}^{0}+u_{1}^{0}\right)
$$

$$
\frac{u_{2}^{1}-u_{2}^{0}}{\Delta t}=\frac{c}{2 \Delta x}\left(u_{1}^{0}-u_{3}^{0}\right)+\frac{c^{2} \Delta t}{2 \Delta x^{2}}\left(u_{3}^{0}-2 u_{2}^{0}+u_{1}^{0}\right) \quad(\text { Lax-Wendroff method })
$$

Now, if we let $r=0.5$ and $c=1$, then $u_{2}^{1}-u_{2}^{0}=-\frac{1}{4}\left(u_{3}^{0}-u_{1}^{0}\right)+\frac{1}{8}\left(u_{3}^{0}-2 u_{2}^{0}+u_{1}^{0}\right)$.

We consider now a high-order interpolation, i.e. higher than quadratic. Proceeding in the same way in part above by using 4 points, in order to get a cubic interpolant. Using $\left(x_{0}, u_{0}^{0}\right),\left(x_{1}, u_{1}^{0}\right),\left(x_{2}, u_{2}^{0}\right)$ and $\left(x_{3}, u_{3}^{0}\right)$ we have

$$
\begin{aligned}
L(x)= & u_{0}^{0} l_{0}(x)+u_{1}^{0} l_{1}(x)+u_{2}^{0} l_{2}(x)+u_{3}^{0} l_{3}(x) \\
= & u_{0}^{0} \frac{\left(x-x_{1}\right)\left(x-x_{2}\right)\left(x-x_{3}\right)}{-6 \Delta x^{3}}+u_{1}^{0} \frac{\left(x-x_{0}\right)\left(x-x_{2}\right)\left(x-x_{3}\right)}{2 \Delta x^{3}} \\
& +u_{2}^{0} \frac{\left(x-x_{0}\right)\left(x-x_{1}\right)\left(x-x_{3}\right)}{-2 \Delta x^{3}}+u_{3}^{0} \frac{\left(x-x_{0}\right)\left(x-x_{1}\right)\left(x-x_{2}\right)}{6 \Delta x^{3}} .
\end{aligned}
$$

therefore, 
finally,

$$
\begin{aligned}
u_{2}^{1} \approx & L\left(x_{2}-c \Delta t\right)=u_{2}^{0}+\frac{c \Delta t}{6 \Delta x}\left(-u_{0}^{0}+6 u_{1}^{0}-3 u_{2}^{0}-2 u_{3}^{0}\right)+\frac{c^{2} \Delta t^{2}}{6 \Delta x^{2}}\left(3 u_{1}^{0}-6 u_{2}^{0}+3 u_{3}^{0}\right) \\
& +\frac{c^{3} \Delta t^{3}}{6 \Delta x^{3}}\left(u_{0}^{0}-3 u_{1}^{0}+3 u_{2}^{0}-u_{3}^{0}\right)
\end{aligned}
$$

$$
\begin{aligned}
\frac{u_{2}^{1}-u_{2}^{0}}{\Delta t}= & \frac{c}{6 \Delta x}\left(-u_{0}^{0}+6 u_{1}^{0}-3 u_{2}^{0}-2 u_{3}^{0}\right)+\frac{c^{2} \Delta t}{2 \Delta x^{2}}\left(u_{3}^{0}-2 u_{2}^{0}+u_{1}^{0}\right) \\
& +\frac{c^{2} \Delta t^{2}}{6 \Delta x^{2}}\left(3 u_{1}^{0}-6 u_{2}^{0}+3 u_{3}^{0}\right)+\frac{c^{2} \Delta t^{2}}{6 \Delta x^{3}}\left(u_{1}^{0}-3 u_{1}^{0}+3 u_{2}^{0}-u_{3}^{0}\right) .
\end{aligned}
$$

Now, we can see the Stability as follows. Performing Von Neumann gives the following,

$$
\begin{aligned}
G(k)= & +\frac{r}{6}\left(-\mathrm{e}^{-2 k \Delta k}+6 \mathrm{e}^{-k \Delta k}-3-2 \mathrm{e}^{k \Delta k}\right)+\frac{r^{2}}{6}\left(3 \mathrm{e}^{-k \Delta k}-6+3 \mathrm{e}^{k \Delta k}\right) \\
& +\frac{r^{3}}{6}\left(\mathrm{e}^{-2 k \Delta k}-3 \mathrm{e}^{-2 k \Delta k}+3-\mathrm{e}^{k \Delta k}\right)
\end{aligned}
$$

In order to find out for which values of $r$ the scheme is stable, here we plotted $|G(k)|$ for various values of $r$, ranging from $r=0$ and $r=1$ since that CFL condition for the other schemes was $r \leq 1$, the results shown on Figure 9 (plot of $|G(k)|$ against $k / \Delta x$ for $r=0: 0.02: 1$, the top straight line corresponds to $r=0$ and therefore $|G(k)| r_{2}<|G(k)| r_{1}$ for $r_{1}<r_{2}$ pointwise), indicate that $r \leq 1$ ensures stability, since $|G(k)| \leq 1$ for all $k \in[0,2 \pi / \Delta x]$.

For the accuracy we compute the LTE of the scheme,

$$
\begin{aligned}
\xi(\Delta x, \Delta t)= & \frac{1}{\Delta t}(u(x, t+\Delta t)-u(x, t)) \\
& -\left[\frac{c}{6 \Delta x}(-u(x-2 \Delta x, t)+6 u(x-\Delta x, t)-3 u(x, t)-2 u(x+\Delta x, t))\right. \\
& +\frac{c^{2} \Delta t}{6 \Delta x^{2}}(3 u(x-\Delta x, t)-6 u(x, t)+3 u(x+\Delta x, t)) \\
& \left.+\frac{c^{3} \Delta t^{2}}{6 \Delta x^{3}}(u(x-2 \Delta x, t)--3 u(x-\Delta x, t)+3 u(x, t)-(x+\Delta x, t))\right] . \\
= & \frac{1}{\Delta t}\left((\Delta t) u_{t}+\frac{\Delta t^{2}}{2} u_{t t}+\frac{\Delta t^{3}}{6} u_{t t t}+\frac{\Delta t^{4}}{24} u_{t t t t}\right) \\
& -\left[\frac{c}{6 \Delta x}\left(-6(\Delta x) u_{x}-\frac{1}{2}\left(\Delta x^{4}\right) u_{x x x x}+\frac{1}{5}\left(\Delta x^{5}\right) u_{x x x x x}-\frac{1}{12}\left(\Delta x^{6}\right) u_{x x x x x x}\right)\right. \\
& +\frac{c^{2} \Delta t}{6 \Delta x^{2}}\left(-3\left(\Delta x^{2}\right) u_{x x}+\frac{1}{4}\left(\Delta x^{4}\right) u_{x x x x}+\frac{1}{120}\left(\Delta x^{6}\right) u_{x x x x x x}\right) \\
& +\frac{c^{3} \Delta t^{2}}{6 \Delta x^{3}}\left(-\left(\Delta x^{3}\right) u_{x x x}+\frac{1}{2}\left(\Delta x^{4}\right) u_{x x x x}-\frac{1}{40}\left(\Delta x^{5}\right) u_{x x x x x}+\frac{1}{12}\left(\Delta x^{6}\right) u_{x x x x x x}\right) . \\
= & (\underbrace{u_{t}+c u_{x}}_{=0})+\frac{\Delta t}{2}(\underbrace{u_{t t}-c^{2} u_{x x}}_{=0})+\frac{\Delta t^{2}}{6}(\underbrace{u_{t t t}+c^{3} u_{x x x}}_{=0}) \\
& +\frac{\Delta t^{3}}{24} u_{t t t t}+\frac{c \Delta t^{3}}{12}\left(u_{x x x x}-\frac{c^{2}}{6} u_{x x x x x x}\right) \\
= & O\left((\Delta t)^{3}-(\Delta x)^{3}\right)
\end{aligned}
$$


Therefore, if we use the CFL condition $r \leq 1$, we expect the GTE to be $O\left((\Delta t)^{3}\right)$.

We can see the results for $u_{0}(x)=\cos x$ where the convergence is shown on Figure 10. Here the grid resolution was varied from $\mathrm{d} x=2 \pi / 2^{5}$ to $\mathrm{d} x=2 \pi / 2^{12}$. Finally, for $u_{0}(x)=\operatorname{box}(x-t)$ the convergence results are shown on Figure 11, where the grid resolution was varied from $\mathrm{d} x=2 \pi / 2^{4}$ to $\mathrm{d} x=2 \pi / 2^{16}$.

Note. As for Lax-Friedrich and Lax-Wendroff, those results should not be surprising, because we cannot expect a method based on the Taylor series of a function to work for a $C^{0}$ function.

We can see the box evolve when advected by a 3rd order scheme. This is presented in a series of snapshots put together in Figure 12. Although this scheme fails to capture the discontinuities of the function, just like the previous schemes, the oscillations created near the discontinuities don't seem to grow very fast. Moreover, the overall shape of the box is (at least visually) better preserved that when advected with L-F of L-W, which provides a simple explanation as to why the rate of convergence of the $L^{1}$ norm is better than that with those schemes.
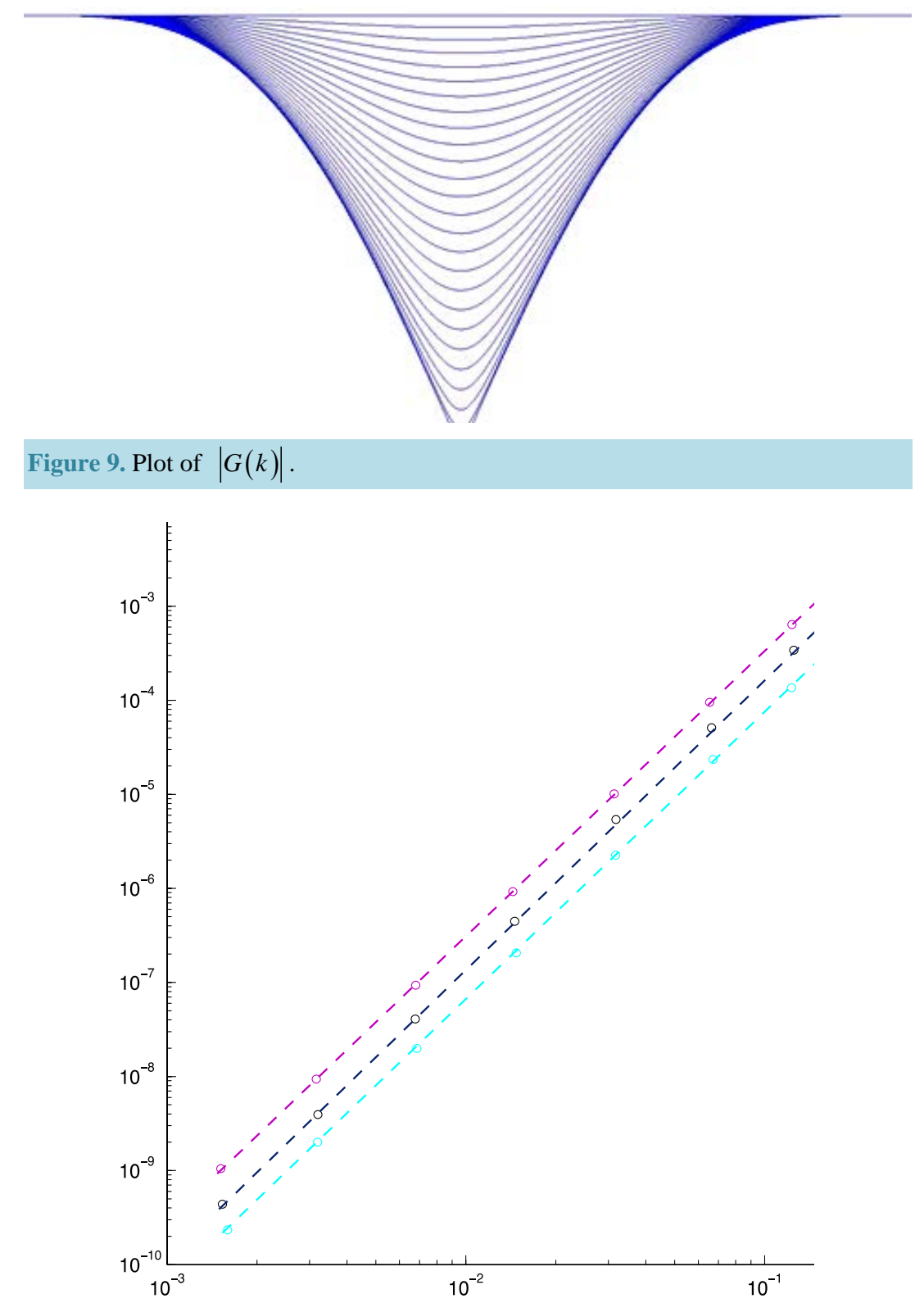

Figure 10. Convergence plot for $u(x, t)=\cos (x-t)$ advected using a 3rd order scheme. 


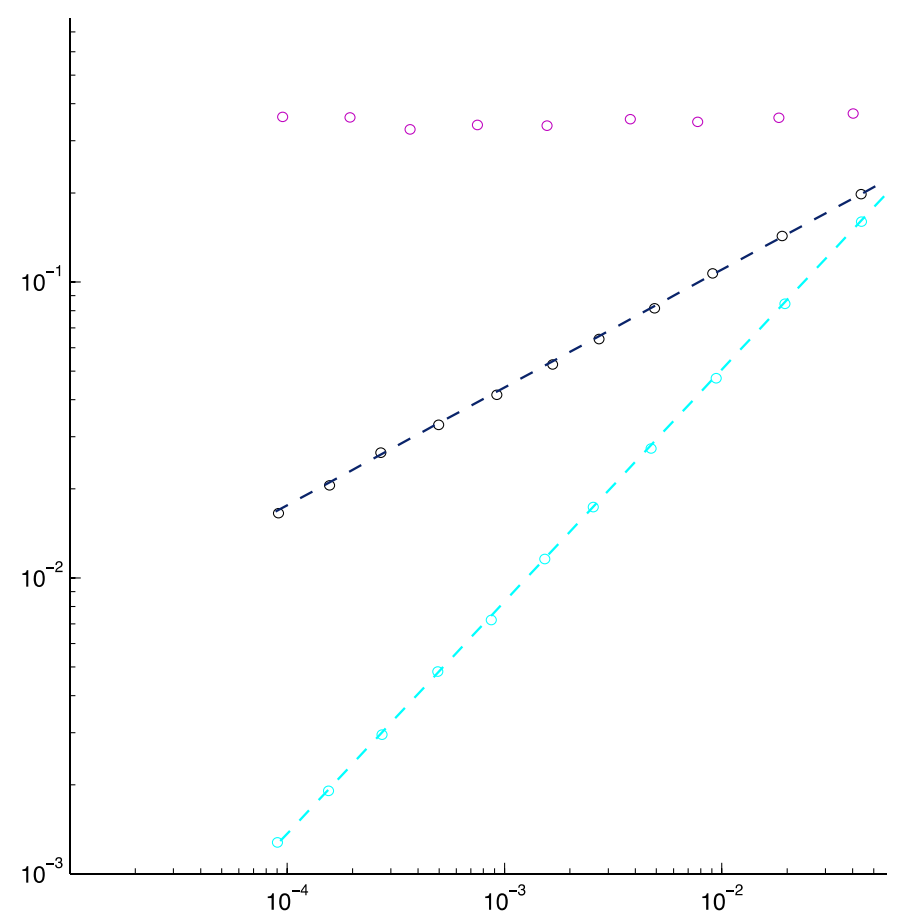

Figure 11. Convergence plot for $u(x, t)=\operatorname{box}(x-t)$ advected using a 3rd order scheme.

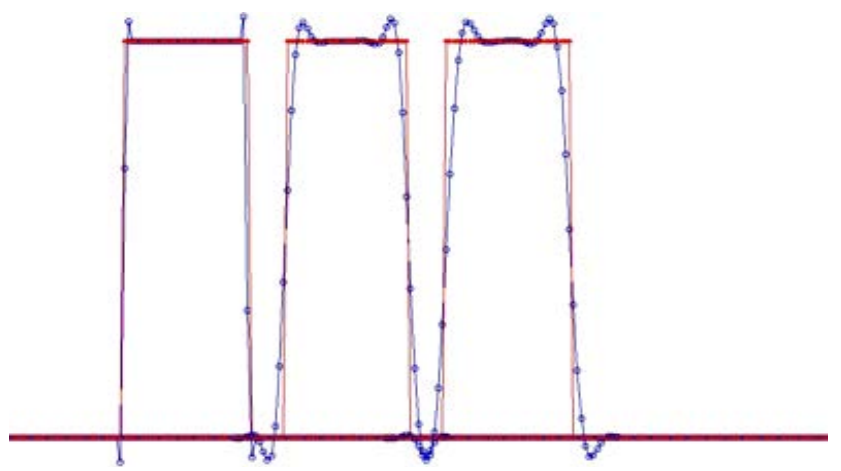

Figure 12. Snapshots for $u(x, t)=\operatorname{box}(x-t)$ advected by a

3rd order scheme.

\section{Acknowledgements}

I would like to thank the referee for his valuable suggestions that improved the presentation of this paper and my gratitude to Department of Mathematics of the Universidad Tecnológica de Pereira (Colombia) and the group GEDNOL.

\section{References}

[1] Strikwerda, J.C. (1989) Finite Difference Schemes and Partial Differential Equations. Wadsworth \& Brooks, USA.

[2] McRea, G.J. and Godin, W.R. (1967) Numerical Solution of Atmospheric Diffusion for Chemically Reacting Flows. Journal of Computational Physics, 77, 1-42.

[3] Morton, K.W. (1980) Stability of Finite Difference Approximations to a Diffusion-Convection Equation. International Journal for Numerical Methods in Engineering, 15, 677-683. http://dx.doi.org/10.1002/nme.1620150505

[4] Hundsdorfer, W. and Koren, B. (1995) A Positive Finite-Difference Advection Scheme Applied on Locally Refined 
Grids. Journal of Computational Physics, 117, 35-36. http://dx.doi.org/10.1006/jcph.1995.1042

[5] Canuto, C. and Hussaini, M. (1988) Spectral Methods in Fluids Dynamics. Springer Series in Computational Physics, Springer-Verlag, Berlin. http://dx.doi.org/10.1007/978-3-642-84108-8

[6] Mitchell, A.R. and Griffiths, D.F. (1980) The Finite Difference Method in Partial Differential Equations. John Wiley \& Sons, Chichester.

[7] Mickens, R.E. (2000) Applications of Nonstandard Finite Differences Schemes. World Scientific Publishing, River Edge.

[8] Dehghan, M. (2005) On the Numerical Solution of the One-Dimensional Convection-Diffusion Equation. Mathematical Problems in Engineering, 1, 61-74. http://dx.doi.org/10.1155/MPE.2005.61 
Scientific Research Publishing (SCIRP) is one of the largest Open Access journal publishers. It is currently publishing more than 200 open access, online, peer-reviewed journals covering a wide range of academic disciplines. SCIRP serves the worldwide academic communities and contributes to the progress and application of science with its publication.

Other selected journals from SCIRP are listed as below. Submit your manuscript to us via either submit@scirp.org or Online Submission Portal.
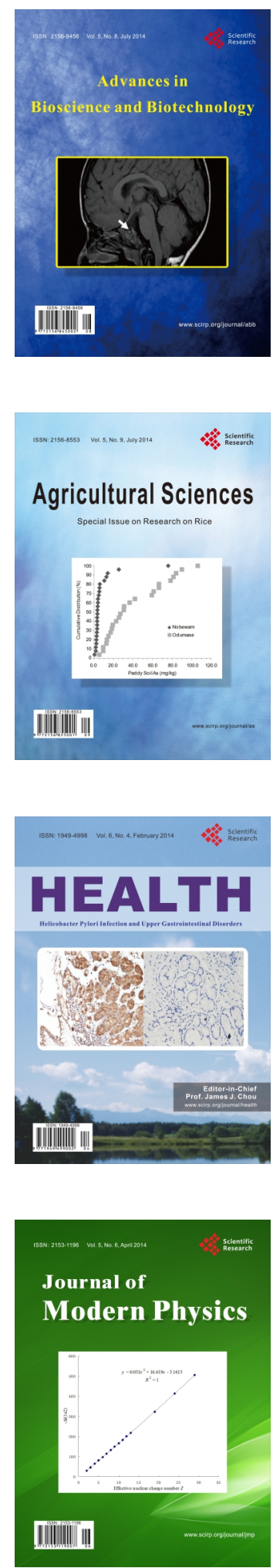
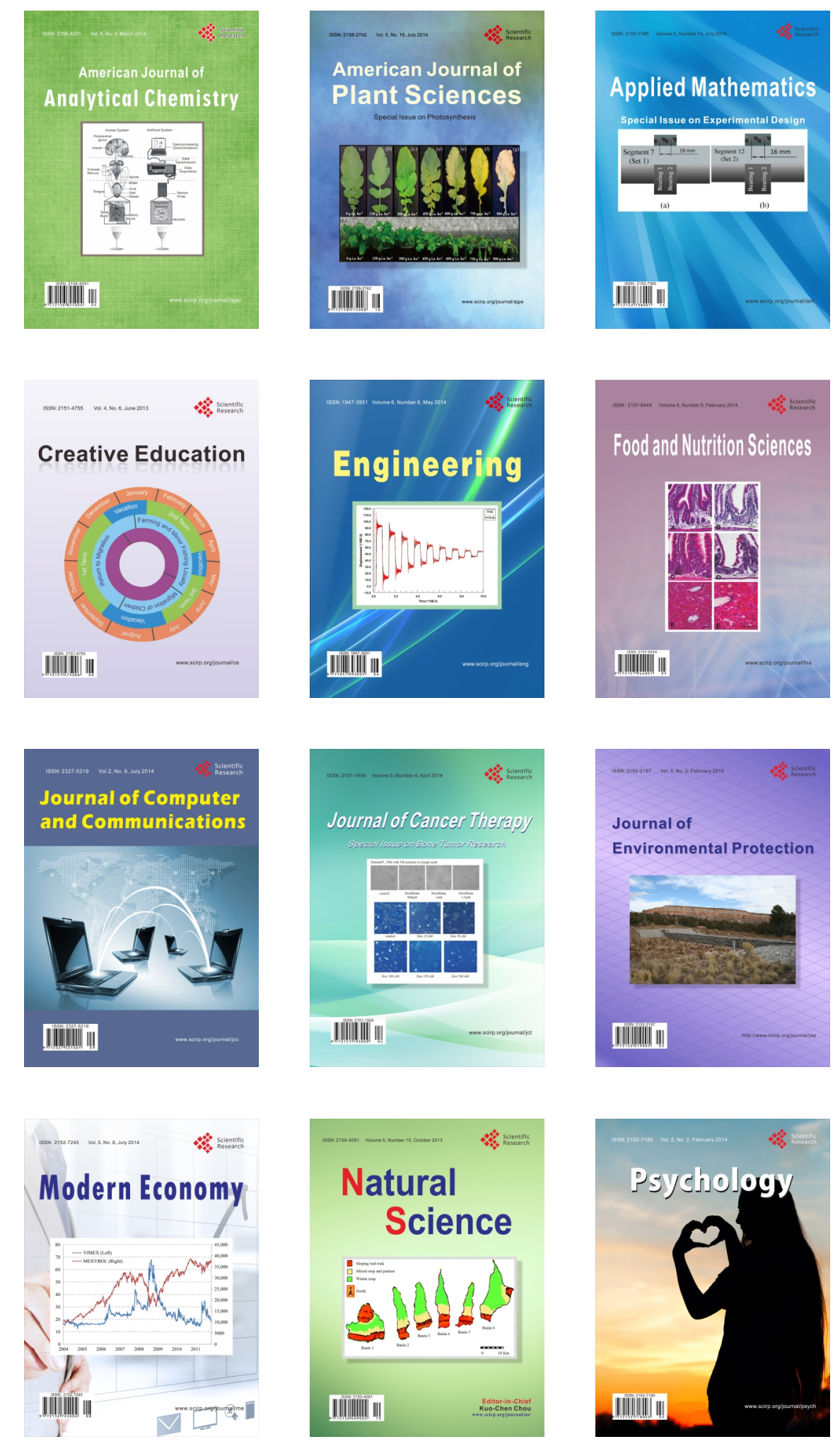